\title{
Combination of ${ }^{68} \mathrm{Ga}-\mathrm{PSMA}$ PET/CT and Multiparametric MRI Improves the Detection of Clinically Significant Prostate Cancer: A Lesion-by-Lesion Analysis
}

\author{
Mengxia Chen*1, Qing Zhang*1, Chengwei Zhang*1, Xiaozhi Zhao ${ }^{1}$, Giancarlo Marra ${ }^{2}$, Jie Gao ${ }^{1}$, Xiaoyu Lv¹, \\ Bing Zhang ${ }^{3}$, Yao $\mathrm{Fu}^{4}$, Feng Wang ${ }^{5}$, Xuefeng Qiu ${ }^{1}$, and Hongqian Guo ${ }^{1}$ \\ ${ }^{I}$ Department of Urology, Drum Tower Hospital, Medical School of Nanjing University, Institute of Urology, Nanjing University, \\ Jiangsu, China; ${ }^{2}$ Department of Urology, San Glovanni Battista Hospital, Città della Salute Scienza and University of Turin, Turin, \\ Italy; ${ }^{3}$ Department of Radiology, Drum Tower Hospital, Medical School of Nanjing University, Nanjing, Jiangsu, China; ${ }^{4}$ Department \\ of Pathology, Drum Tower Hospital, Medical School of Nanjing University, Nanjing, Jiangsu, China; and ${ }^{5}$ Department of Nuclear \\ Medicine, Nanjing First Hospital, Nanjing Medical University, Nanjing, Jiangsu, China
}

Our purpose was to explore whether ${ }^{68} \mathrm{Ga}-\mathrm{PSMA} \mathrm{PET} / \mathrm{CT}$ alone (PET/CT) or in combination with multiparametric MRI (PET/MRI) can improve the detection of clinically significant prostate cancer (PCa). Methods: We retrospectively enrolled 54 patients who underwent both $\mathrm{MRI}$ and PET/CT before radical prostatectomy. Regions of interest on MR images, PET/CT images, and pathologic images were marked. A lesion was defined as a region of interest marked on images obtained with any of the 3 modalities. All lesions were characterized using the prostate imaging reporting and data system (PI-RADS), the molecular imaging PSMA expression score, and the pathologic results and analyzed. Diagnostic performance was analyzed by receiver-operating-characteristic analysis. Specific improvement for lesions with different PI-RADS scores was analyzed using the net reclassification index (NRI). Results: In total, 90 lesions from 54 patients were analyzed, among which 66 lesions represented clinically significant PCa. Receiver-operating-characteristic analysis showed PET/MRI to perform better than MRI in detecting clinically significant PCa (change in area under the curve, 0.06; 95\% confidence interval, $0.01-0.12 ; P<0.05)$. With the calculated cutoff, PET/MRI performed significantly better than MRI (NRI, 21.9\%; $P<$ $0.01)$, with an improvement in sensitivity $(89 \%$ vs. $76 \%, P<0.01)$ at no sacrifice of specificity ( $96 \%$ vs. $88 \%, P>0.05)$. Improvement in diagnosing clinically significant $\mathrm{PCa}$ occurred for lesions classified as PI-RADS 3 (NRI, 66.7\%; $P<0.01$ ). Conclusion: PET/MRI improves the detection of clinically significant PCa for PI-RADS 3 lesions.

Key Words: multiparametric MRI; ${ }^{68} \mathrm{Ga}-\mathrm{PSMA} \mathrm{PET} / \mathrm{CT}$; combination; clinically significant prostate cancer

J Nucl Med 2019; 60:944-949

DOI: 10.2967/jnumed.118.221010

\footnotetext{
Received Sep. 26, 2018; revision accepted Nov. 26, 2018.

For correspondence or reprints contact either of the following: Hongqian Guo, Department of Urology, Drum Tower Hospital, Medical School of Nanjing University, 321 Zhongshan Rd., Gulou District, Nanjing 210008, China.

E-mail: dr.ghq@nju.edu.cn

Xuefeng Qiu, Department of Urology, Drum Tower Hospital, Medical School of Nanjing University, 321 Zhongshan Rd., Gulou District, Nanjing 210008, China.

E-mail: Xuefeng_Qiu@163.com

${ }^{*}$ Contributed equally to this work

Published online Dec. 14, 2018.

COPYRIGHT (C 2019 by the Society of Nuclear Medicine and Molecular Imaging.
}

$\mathbf{E}$ arly detection of clinically significant prostate cancer (PCa) is of great importance for proper patient management $(1,2)$. Systematic transrectal ultrasound biopsy is currently recommended as the standard strategy by international guidelines in cases of suspected PCa $(3,4)$. However, this approach is responsible for the underdetection of clinically significant PCa and the overdetection of clinically insignificant cancer $(5,6)$.

Prostate multiparametric MRI has recently shown promising results for detection of clinically significant PCa compared with systematic biopsy $(7,8)$ and is currently revolutionizing the $\mathrm{PCa}$ diagnostic pathway $(9,10)$. According to recent studies, multiparametric MRI allows the detection of $12 \%-18 \%$ more clinically significant PCa and 5\% less clinically insignificant PCa $(9,10)$. However, the detection of clinically significant PCa on multiparametric MRI is variable and depends on several factors, including tumor volume, Gleason score (GS), and location, and the interpretation of multiparametric MRI is operator-dependent, yielding an obvious learning curve to reach high diagnostic standards $(11,12)$. All these factors cause approximately $12 \%-26 \%$ of clinically significant PCa to be missed $(5,9)$.

${ }^{68} \mathrm{Ga}$-labeled molecular imaging with PET-targeted prostate-specific membrane antigen $\left({ }^{68} \mathrm{Ga}\right.$-PSMA PET) is a relatively new nuclear imaging modality showing high sensitivity and specificity, initially proposed to identify locations of recurrent PCa (13-16). Recently, several studies have investigated the role of ${ }^{68} \mathrm{Ga}-\mathrm{PSMA}$ PET/CT in a first-line diagnostic setting also (17-20), and some have investigated the role of simultaneous PET/MRI in PCa detection, concluding that it may further improve diagnostic accuracy in terms of cancer detection and gross volume estimation (21-24).

Our aim was to assess whether the combination of multiparametric MRI and ${ }^{68} \mathrm{Ga}$-PSMA PET/CT (PET/MRI) can improve the detection rate of clinically significant $\mathrm{PCa}$ compared with multiparametric MRI alone (MRI) or ${ }^{68} \mathrm{Ga}$-PSMA PET/CT alone (PET/CT) in a consecutive cohort of men who underwent subsequent surgical treatment.

\section{MATERIALS AND METHODS}

\section{Patients}

Between November 2017 and June 2018, we retrospectively included 54 consecutive patients who had undergone MRI and PET/CT for suspected PCa and, subsequently, radical prostatectomy because of histologically confirmed PCa. We excluded men who had not given 


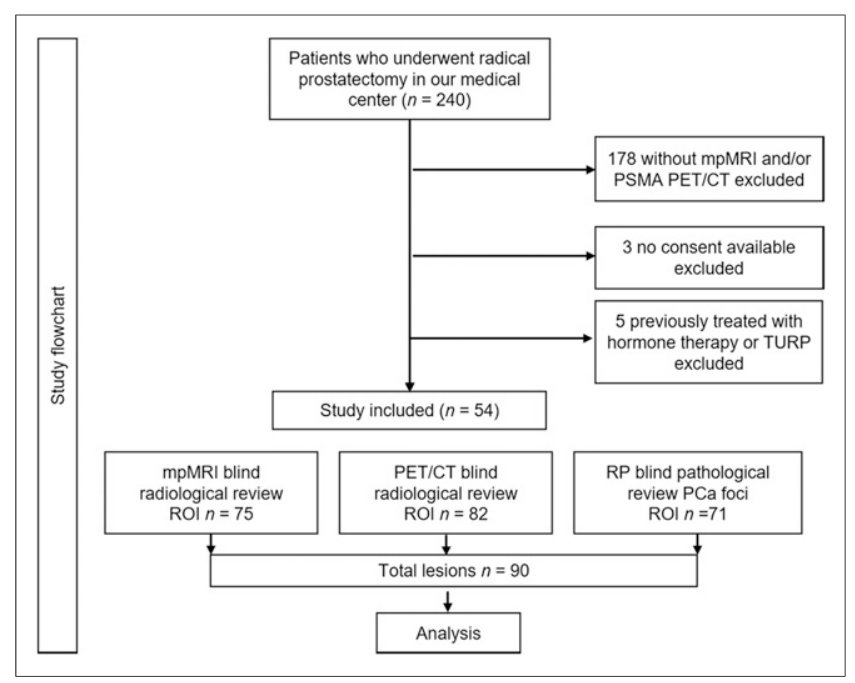

FIGURE 1. Study flowchart showing excluded patients, reason for exclusion, and defined criteria for ROI. $\mathrm{mpMRI}=$ multiparametric MRI; TURP $=$ transurethral resection of the prostate.

written informed consent $(n=3)$ or who had undergone previous treatment (hormone therapy, $n=3$; transurethral resection of the prostate, $n=2$ ) (Fig. 1). The reason for exclusion of patients with hormone therapy $(n=3)$ was that their pathologic results showed a severe treatment response and GS could not be accurately assigned. Finally, 54 patients were analyzed, with a median age of $69 \mathrm{y}$ and a median prostate-specific antigen level of $13.53 \mathrm{ng} / \mathrm{mL}$. The study protocol was approved by the Ethics Committee of the Drum Tower Hospital (approval 2017-147-01).

\section{MRI Examination and Image Evaluation}

All patients underwent pelvic MRI using an Achieva 3.0-T TX scanner (Philips) with a 16-channel phased-array coil as described previously (25). Transverse, coronal, and sagittal T2-weighted turbo spin-echo images were acquired (18 slices $3 \mathrm{~mm}$ thick with an intersection gap of $0.5 \mathrm{~mm}$; repetition time, $3,744 \mathrm{~ms}$; echo time, 120 $\mathrm{ms}$; number of signal averages, 2; resolution, $1.49 \times 1.51 \mathrm{~mm})$. Also acquired were diffusion-weighted spin-echo echo-planar images (18 slices $3 \mathrm{~mm}$ thick with an intersection gap of $1 \mathrm{~mm}$; repetition time, $925 \mathrm{~ms}$; echo time, $41 \mathrm{~ms}$; number of signal averages, 1; resolution, $3 \mathrm{~mm} \times 3 \mathrm{~mm}$; b-factor, $0 / 800 / 1,500 \mathrm{~s} / \mathrm{mm}^{2}$ ), as well as dynamic contrast-enhanced T1-weighted high-resolution isotropic volume images with fat suppression after gadolinium injection (133 slices $3 \mathrm{~mm}$ thick with no intersection gap; repetition time, $3.1 \mathrm{~ms}$; echo time, 1.46 $\mathrm{ms}$; number of signal averages, 1 ; resolution, $1.49 \times 1.51 \mathrm{~mm}$; dynamic scan time, 00:06.9). All MRI images were reviewed by a dedicated radiologist (over 10 y of prostate MRI experience), who was masked to the MRI and pathologic results. Regions of interest (ROIs), defined as regions with abnormal signal on MR images, were contoured and scored using version 2 of the prostate imaging reporting and data system (PI-RADS) (26).

\section{PET/CT Examination and Image Evaluation}

${ }^{68} \mathrm{Ga}$-PSMA-11 was synthesized using an ITG semiautomated module as described previously (27). One hour before scanning, all patients were intravenously injected with ${ }^{68} \mathrm{Ga}-\mathrm{PSMA}-11$ (median, 131.72 MBq; range, 130.6-177.6 MBq). PET/CT was performed on a uMI 780 PET/CT scanner (United Imaging Healthcare). A CT scan (130 keV, $80 \mathrm{mAs})$ and a static emission scan, corrected for dead time, scatter, and decay, were acquired from the vertex to the proximal legs. PET/CT imaging was independently evaluated by a double-trained board-certified nuclear medicine physician (over $10 \mathrm{y}$ of PET/CT experience), who was masked to the MRI and pathologic results. ROIs on PET/CT were defined as an area of uptake in the prostate gland higher than background uptake. ROIs on PET/CT were scored by a molecular imaging PSMA expression score (MI-ES) on a molecular imaging TNM system (28). In brief, by comparison of the uptake level against index organs, MI-ES was divided into a 4-point scale: 0 , below blood pool; 1 , equal to or above blood pool and lower than liver; 2, equal to or above liver and lower than parotid gland; 3 , equal to or above parotid gland.

\section{Whole-Mount Pathologic Evaluation}

After robot-assisted radical prostatectomy, whole-mount tissue was fixed in $10 \%$ formalin, paraffin-embedded, microtome-cut into 4- to 5mm slices, and stained with hematoxylin-eosin according to the Stanford protocol (29). All whole-mount histology slides were subsequently digitalized by a scanning system (NanoZoomer Digital Pathology). All pathologic images were interpreted by a dedicated genitourinary pathologist (over 10 y of experience). To identify pathologic ROIs, each tumor lesion was contoured and a corresponding GS was assigned. Tumor or lesion volume was determined by multiplying the sum of the areas by section thickness $(3 \mathrm{~mm})$ and by a 1.5 correction factor for tissue shrinkage during processing, as performed by others (30). Clinically significant PCa was defined as a cancer volume of at least $0.5 \mathrm{~cm}^{3}$, a GS of at least $3+4$, or a stage of at least pT3 $(31,32)$.

\section{Marking and Scoring of Lesions}

MRI, PET/CT, and pathologic images at the same level of the prostate were matched by a radiologist and genitourinary pathologist according to slice number. ROIs were marked in blue. A lesion was defined as an ROI marked on images obtained with any of the 3 modalities. The corresponding ROI was marked in red on images that did not show this lesion (Fig. 2). Afterward, in a masked manner, all lesions were scored using PI-RADS, MI-ES, and GS, and tumor volume was recorded (Fig. 1). For PET/MRI, a score was calculated using PI-RADS plus MI-ES. As a result, a PI-RADS/MI-ES score could reflect the possibility of clinically significant $\mathrm{PCa}$ not only by morphology on MRI but also by PSMA expression status on PET/CT.

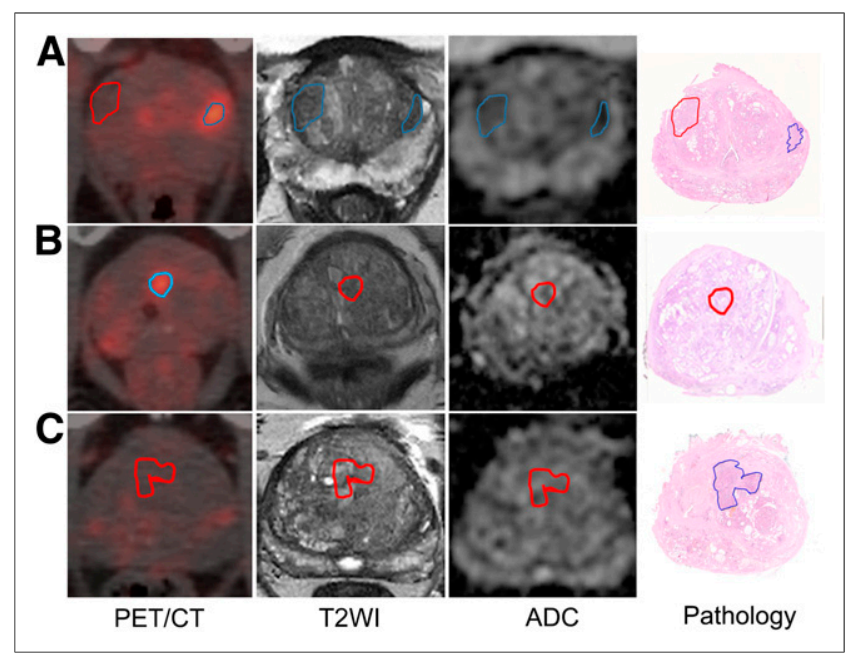

FIGURE 2. Marking of lesions. (A) Example of one lesion that was identified on all types of images (right blue ROI) and a second lesion that was identified only on MRI (left blue ROI). Corresponding ROI for the second lesion on PET/CT and pathology is marked in red. (B) Example of a lesion that was identified only on PET/CT (blue ROI); corresponding $\mathrm{ROI}$ on MRI and pathology is marked in red. (C) Example of a lesion that was identified only on pathology (blue ROI); corresponding $\mathrm{ROI}$ on $\mathrm{PET} / \mathrm{CT}$ and $\mathrm{MRI}$ is marked in red. $\mathrm{ADC}=$ apparent diffusion coefficient map; T2WI = T2-weighted MRI. 


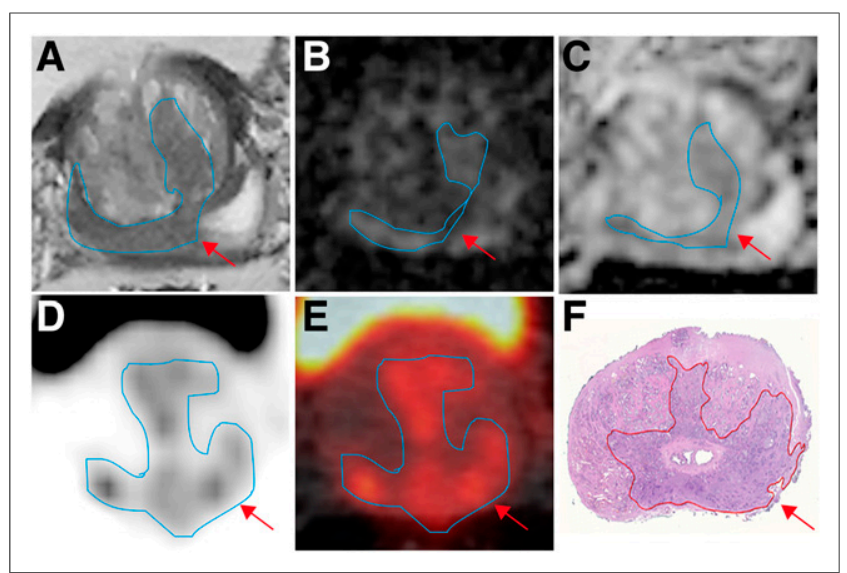

FIGURE 3. A 66-y-old patient with prostate-specific antigen level of $7.89 \mathrm{ng} / \mathrm{mL}$. (A) Transverse T2-weighted MR image shows lesion with noncircumscribed moderate hypointensity in peripheral zone and transition zone (arrow). (B) Diffusion-weighted imaging with b-factor of 1,500 shows moderately high signal at edge of peripheral zone and central part of left transition zone (arrow). (C) Apparent-diffusion-coefficient map shows linear moderate hypointensity in central part of right peripheral zone and left transition zone (arrow). All findings result in PI-RADS 3. ( $D$ and E) PET shows intense focal uptake in peripheral zone and transition zone (arrow) that is higher than liver, resulting in MI-ES 2. (F) Whole-mount histology proving that lesion seen on PET/CT is clinically significant PCa with GS $4+5=9$ and total tumor volume of $0.21 \mathrm{~cm}^{3}$.

Figure 3 shows an example of a lesion scored as PI-RADS 3 and MI-ES 2. Lesions on the final histologic specimen were considered the gold standard to define presence or absence of significant or insignificant PCa.

\section{Statistical Analysis}

Diagnostic performance for clinically significant PCa on MRI, $\mathrm{PET} / \mathrm{CT}$, and PET/MRI was assessed using area under the receiveroperating-characteristic curve (AUC), and 95\% confidence interval (95\% CI) was calculated as proposed by Obuchowski (33). Logistic generalized-estimating-equation models were applied to estimate sensitivity, specificity, and 95\% CI (34-36). Differences in sensitivity and specificity were analyzed using the McNemar test. A net reclassification index (NRI) (37) was calculated for lesions, with the calculated cutoff comparing MRI with PET/MRI. Briefly, NRI presents the net percentage of lesions with the event of interest correctly classified upward. A significance level of 5\% was used. All analyses were performed using SPSS software, version 22.0 (IBM Corp.).

\section{RESULTS}

\section{Patient Characteristics and Imaging Findings}

In total, 54 patients were included (Fig. 1). Table 1 shows their characteristics. All patients underwent MRI first, followed by prostate biopsy and PET/CT. The median intervals were $5 \mathrm{~d}$ (range, 3-9 d) between MRI and biopsy, 22 d (range, 14-34 d) between biopsy and PET/CT, and $28 \mathrm{~d}$ (range, 23-39 d) between MRI and PET/CT. Ninety lesions were identified and matched on MRI, PET/CT, and pathology, among which 66 were proved to be clinically significant PCa, with 6.1\% (4/66) being GS 6, 62.1\% (41/ 66) GS 7, and $31.8 \%(21 / 66)$ GS 8 or higher.

\section{Diagnostic Performance of MRI, PET/CT, and PET/MRI}

AUC of PET/MRI Outperformed MRI and PET/CT. Figure 4 and Table 2 show the AUC and 95\% CI of each scan for performance in diagnosing clinically significant PCa compared with the final
TABLE 1

Baseline Features of Included Cases $(n=54)$

\begin{tabular}{lc}
\hline \multicolumn{1}{c}{ Characteristic } & Value \\
\hline Age $(\mathrm{y})$, median & 69 (range, 55-84) \\
\hline Tumor volume, median & 1.02 (range, 0.03-20.50) \\
\hline Initial PSA (ng/dL), median & 13.53 (range, 4.04-110.00) \\
\hline GS $(n)$ & \\
\hline $3+3=6$ & $6(11.1 \%)$ \\
$3+4=7$ & $13(24.1 \%)$ \\
$4+3=7$ & $16(29.6 \%)$ \\
\hline $4+4=8 / 5+3=8$ & $9(16.7 \%)$ \\
$9-10$ & $10(18.5 \%)$ \\
\hline pT stage $(n)$ & $17(31.2 \%)$ \\
\hline 2 & $25(46.3 \%)$ \\
\hline $3 a$ & $12(22.2 \%)$ \\
\hline $3 b$ & $0(0 \%)$ \\
\hline 4 & \\
\hline PSA $=$ prostate-specific antigen level. \\
\hline
\end{tabular}

pathologic results. The AUC of PET/MRI showed an improvement in diagnosis of clinically significant PCa over MRI alone (change in AUC, 0.06 ; 95\% CI, 0.01-0.12; $P<0.05$ ) or PET/CT alone (change in AUC, 0.09; 95\% CI, 0.03-0.16; $P<0.05$ ). No significant difference in AUC was seen between MRI and PET/CT (change in AUC, $0.03 ; 95 \% \mathrm{CI},-0.08$ to $0.14 ; P=0.59$ ).

PET/MRI Had Higher Sensitivity Than MRI and Higher Specificity Than PET/CT. The Youden-selected threshold, sensitivity, and specificity of each type of imaging are listed in Table 2.

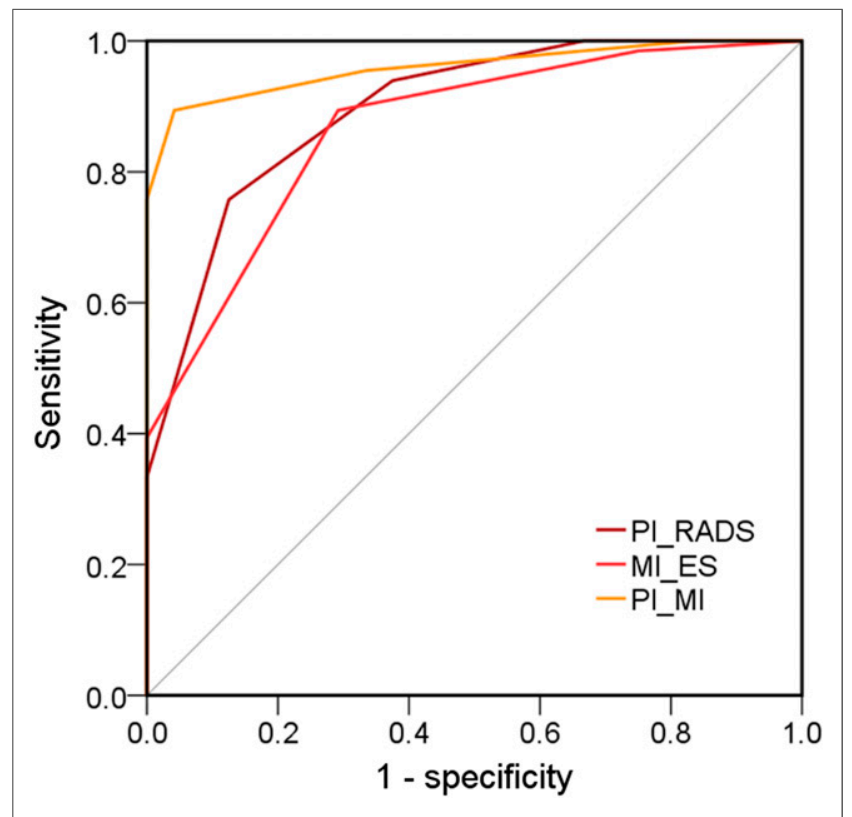

FIGURE 4. Receiver-operating-characteristic analysis of MRI, PET/CT, and PET/MRI for detection of clinically significant PCa. PI_MI = PIRADS/MI-ES. 
TABLE 2

Diagnostic Accuracy for Clinically Significant PCa Using MRI, PET/CT, and PET/MRI Compared with Pathologic Results

\begin{tabular}{|c|c|c|c|c|}
\hline Modality & AUC & Youden-selected threshold & Sensitivity (\%) at threshold & Specificity (\%) at threshold \\
\hline MRI & $0.90(95 \% \mathrm{Cl}, 0.81-0.95)^{\star}$ & 4 & $76(95 \% \mathrm{Cl}, 64-86)^{\star \dagger}$ & $88(95 \% \mathrm{Cl}, 68-97)$ \\
\hline $\mathrm{PET} / \mathrm{CT}$ & $0.87(95 \% \mathrm{Cl}, 0.78-0.93)^{\ddagger}$ & 2 & $89(95 \% \mathrm{Cl}, 79-96)^{\dagger}$ & $71(95 \% \mathrm{Cl}, 49-87)^{\ddagger}$ \\
\hline PET/MRI & $0.96(95 \% \mathrm{Cl}, 0.90-0.99)^{\star \ddagger}$ & 5 & $89(95 \% \mathrm{Cl}, 79-96)^{\star}$ & $96(95 \% \mathrm{Cl}, 79-100)^{\ddagger}$ \\
\hline \multicolumn{5}{|c|}{$\begin{array}{l}{ }^{*} \mathrm{MRI} \text { vs. } \mathrm{PET} / \mathrm{MRI}, P<0.05 \\
{ }^{\dagger} \mathrm{MRI} \text { vs. } \mathrm{PET} / \mathrm{CT}, P<0.05 \\
{ }^{\mathrm{F}} \mathrm{PET} / \mathrm{CT} \text { vs. } \mathrm{PET} / \mathrm{MRI}, P<0.05 .\end{array}$} \\
\hline
\end{tabular}

The cutoffs calculated by the Youden-selected threshold for MRI, $\mathrm{PET} / \mathrm{CT}$, and PET/MRI for clinically significant PCa were a PIRADS of at least 4, an MI-ES of at least 2, and a PI-RADS/MI-ES of at least 5 , respectively. Diagnostic results corrected by the actual pathologic results are listed in Table 3. With the calculated cutoff, the sensitivities of MRI, PET/CT, and PET/MRI were $76 \%$ (95\% CI, 64-86), 89\% (95\% CI, 79-96), and 89\% (95\% CI, 7996), respectively, and the specificities were $88 \%$ (95\% CI, 68-97), 71\% (95\% CI, 49-87), and 96\% (95\% CI, 79-100), respectively. PET/MRI showed a higher sensitivity than MRI (95\% CI for difference, 5-22; $P<0.01$ ), without sacrificing specificity $(95 \%$ CI for difference, -2 to $19 ; P>0.05)$. With the calculated cutoff, PET/MRI had a significantly positive NRI compared with MRI (NRI, 21.9\%; $P<0.01$ ). Although PET/CT had a significantly higher sensitivity than MRI (95\% CI for difference, $4-23 ; P<$ 0.01 ), a lower specificity was also observed (95\% CI, -42 to 8 ; $P>0.05)$, though without statistical significance. As a result, no significant overall improvement was seen on PET/CT compared with MRI (NRI, $-3 \%$; $P=0.83$ ). Notably, PET/MRI had a significant improvement in specificity $(95 \% \mathrm{CI}, 4-46 ; P<0.05)$ compared with PET/CT.

PET/MRI Improved the Diagnostic Performance for PI-RADS 3 Lesions. To explore the degree to which the improved diagnostic performance of PET/MRI affected lesions of varying PI-RADS on MRI, we analyzed the NRI results (Table 4). There was a significant diagnostic improvement for lesions classified as PI-RADS 3 on MRI (NRI, 66.7\%; $P<0.05$ ) but not for lesions classified as PI-RADS 2 (NRI, 25\%; $P=0.32$ ), PI-RADS 4 (NRI, 66.7\%; $P=$ 0.16 ), PI-RADS 1 (NRI, 0), or PI-RADS 5 (NRI, 0). Supplemental Table 1 shows the influence of PET/MRI on false-positivity and

TABLE 3

Diagnostic Results of MRI, PET/CT, and PET/MRI on All Lesions with Calculated Cutoff: Classification by Final Pathologic Results

\begin{tabular}{lcccccc}
\hline & \multicolumn{2}{c}{$\begin{array}{c}\text { Clinically } \\
\text { significant PCa }\end{array}$} & & \multicolumn{2}{c}{$\begin{array}{c}\text { Non-clinically } \\
\text { significant PCa* }\end{array}$} \\
\cline { 2 - 3 } \cline { 5 - 6 } Modality & Positive & Negative & & Positive & Negative \\
\hline MRI & 50 & 16 & & 3 & 21 \\
PET/CT & 59 & 7 & & 7 & 17 \\
PET/MRI & 59 & 7 & & 1 & 23 \\
\hline
\end{tabular}

${ }^{*}$ Both normal prostate tissue and clinically insignificant PCa. false-negativity in the various PI-RADS groups (supplemental materials are available at http://jnm.snmjournals.org). PET/MRI reduced the false-negative rate, compared with MRI, in the PIRADS 2 group (30\% vs. 36.4\%) and the PI-RADS 3 group (40\% vs. $66.7 \%)$ and reduced the false-positive rate in the PI-RADS 4 group $(3.4 \%$ vs. $9.7 \%)$.

\section{DISCUSSION}

To our knowledge, this was the first lesion-by-lesion study indicating that PET/MRI improves the detection of clinically significant PCa over MRI or PET/CT alone. Furthermore, we found that this improvement was due mainly to improved detection of lesions that were scored as PI-RADS 3 on MRI. Compared with MRI alone, additional PET/CT scanning can thus prevent a clinically significant PCa diagnosis from being missed and can allow for early and correct diagnosis of tumors requiring active treatment.

Level 1 evidence recently proved MRI to improve the diagnosis of clinically significant $\mathrm{PCa}$ and to decrease unnecessary biopsies and diagnosis of insignificant PCa $(9,10)$. Notably, compared with systematic transrectal ultrasound biopsy, MRI detected $18 \%$ more cases of clinically significant PCa in the PROMIS study (9) and $12 \%$ more in the PRECISION study (10). Using MRI to triage men might allow $27 \%$ of patients to avoid a primary biopsy and might reduce diagnosis of clinically insignificant cancers by $5 \%$ (9). However, a metaanalysis (38) showed that the accuracy of MRI for detection of clinically significant PCa varies widely between studies (44\%-87\%). The same underestimation of clinically significant PCa was also observed in our study. With a PI-RADS cutoff of 4 or more, MRI missed $24.2 \%$ of the clinically significant PCa. In our study, $66.7 \%$ of PI-RADS 3 lesions represented clinically significant PCa. The possibility of missing the diagnosis of clinically significant PCa in some patients with PI-RADS 3 lesions may contribute to the dilemma of whether patients with PI-RADS 3 lesions should undergo prostate biopsy and may lead to substantial patient mismanagement.

Because application of molecularly targeted imaging can provide functional and molecular information, it is expected that the combination of MRI and PET/CT would improve PCa detection. Eiber et al. found PET/MRI and PET/CT to be more sensitive than MRI in overall cancer detection $(98 \%$ and $92 \%$, respectively, vs. $66 \%$ ) (21). More recently, Hicks et al. reported PET/MRI to be more sensitive than MRI (23), and Park et al. reported a $100 \%$ detection rate for PCa on a patient basis (24). Similarly, our results showed diagnostic improvement in the detection of clinically significant PCa by PET/MRI over MRI alone (change in AUC, 0.06). For a PI-RADS/MI-ES cutoff of at least 5 and an MI-ES cutoff of at least 2, PET/MRI and PET/CT were more sensitive than MRI in 
TABLE 4

Diagnostic Improvement for Detection of Clinically Significant PCa for Lesions with Different PI-RADS by PET/MRI

\begin{tabular}{lccccc}
\hline Parameter & PI-RADS 1 & PI-RADS 2 & PI-RADS 3 & PI-RADS 4 & PI-RADS 5 \\
\hline NRI (\%) & 0 & 25 & 66.7 & 66.7 & 0 \\
$P$ & NA & 0.32 & 0.005 & & NA \\
& & & & & \\
\hline NA $=$ not available. & & & & \\
\hline
\end{tabular}

diagnosing clinically significant PCa (89\% and $89 \%$, respectively, vs. $76 \%$ ). The high sensitivity of PET/CT contributes to the improvement of PET/MRI in detecting clinically significant PCa. ${ }^{68} \mathrm{Ga}$-PSMA PET/CT has proven to have high sensitivity in the biochemical recurrence setting (even with a low prostate-specific antigen level (15)) and high accuracy in detecting primary tumor (AUC, 0.83 (21)). Nonetheless, our study found that PET/CT alone suffered from low specificity $(71 \%)$, causing a $29.2 \%$ overdiagnosis of clinically insignificant PCa. As a result, despite the higher sensitivity of PET/CT, it showed no significant overall improvement in detection of clinically significant PCa (NRI, $-3 \% ; P>$ 0.84), compared with MRI. However, PET/MRI had a higher specificity than PET/CT. Compared with MRI alone, PET/MRI was more sensitive in detecting clinically significant $\mathrm{PCa}$, without sacrificing specificity, resulting in a significant diagnostic improvement of NRI $21.9 \%$.

Despite the improved detection of clinically significant PCa found for PET/MRI, the use of ${ }^{68} \mathrm{Ga}$-PSMA for PET/CT remains expensive and not routinely available. It is helpful to identify the specific population who might benefit from the combination. In this study, we found that NRI was significant for the PI-RADS 3 group (NRI, 66.7\%) and, thus, that the improved detection with PET/MRI occurred for lesions classified as PI-RADS 3. The guidelines for PI-RADS, version 2, say that biopsy may be appropriate for patients with PI-RADS 2 or 3 lesions, depending on factors (e.g., high prostate-specific antigen level, family history) other than MRI results alone (26). Our study showed that additional PET/CT in patients with moderate MRI results (PI-RADS 3) might be helpful in making a decision about prostate biopsy. In a patient who has ${ }^{68} \mathrm{Ga}$-PSMA uptake higher than the level in the liver (MI-ES $\geq 2$ ), PI RADS 3 lesions might represent clinically significant $\mathrm{PCa}$. As an illustration of the possibility that clinically significant PCa might be missed on MRI, one case report showed that the use of ${ }^{68} \mathrm{Ga}$-PSMA PET/CT in a patient negative for PCa on MRI allowed for detection and targeted biopsy of the disease (17). However, our study found that for patients with negative MRI findings (PI-RADS 1 or 2), additional PET/CT scanning was of limited value. Because PET/CT has relatively low specificity in the detection of clinically significant $\mathrm{PCa}$, basing a biopsy decision on negative MRI findings (PI-RADS 1 or 2) but positive PET/CT findings might contribute to unnecessary use of prostate biopsy.

Our study had some limitations. First, we used the final pathologic results as a reference standard. Hence, a selection bias likely occurred because all included men already had proven clinically significant PCa and no patients with negative findings or at low risk were included. Nonetheless, the radical prostatectomy specimen is the most accurate final arbiter to determine presence or absence of $\mathrm{PCa}$ in a per-lesion analysis. Furthermore, our work is among the first to address the diagnostic potential of PET/MRI. Second, although we included every lesion seen on all images whether suspected of representing tumor or not, the number of truenegative lesions might not have been large enough because the overall study sample was limited. Third, the fact that the evaluation on PET/CT was a semiquantification but did not include features such as lesion shape or location may account for the low specificity of PET/CT alone. PET/MRI has shown strength in PCa detection, and whether PET/MRI can be applied as a first-line diagnostic tool for all suspected PCa now needs further evaluation.

\section{CONCLUSION}

Our study showed that PET/MRI can improve the detection of clinically significant PCa in patients with moderate MRI results. These findings may help in decision making regarding patients with moderate MRI results, to avoid the missing of clinically significant PCa. Further prospective studies are warranted to confirm our findings.

\section{DISCLOSURE}

This study was supported by grants from the National Natural Science Foundation of China (81772710, 81572519, and 81602232), the Natural Science Foundation of Jiangsu Province (BK20150112 and BK20150097), the Nanjing Medical Science and Technique Development Foundation (QRX17128), the Nanjing Health Distinguished Youth Fund (JQX16025), and the Project of Invigorating Health Care through Science, Technology, and Education, Jiangsu Provincial Key Medical Discipline (Laboratory) (ZDXKB2016014). No other potential conflict of interest relevant to this article was reported.

\section{ACKNOWLEDGMENTS}

We thank Danyan Li and Xiangshan Fan for their kind work in matching the images.

\section{REFERENCES}

1. Loeb S, Bjurlin MA, Nicholson J, et al. Overdiagnosis and overtreatment of prostate cancer. Eur Urol. 2014;65:1046-1055.

2. Fenton JJ, Weyrich MS, Durbin S, Liu Y, Bang H, Melnikow J. Prostate-specific antigen-based screening for prostate cancer: evidence report and systematic review for the US Preventive Services Task Force. JAMA. 2018;319:1914-1931.

3. Carroll PH, Mohler JL. NCCN guidelines updates: prostate cancer and prostate cancer early detection. J Natl Compr Canc Netw. 2018;16:620-623.

4. Mottet N, Bellmunt J, Bolla M, et al. EAU-ESTRO-SIOG guidelines on prostate cancer. Part 1: screening, diagnosis, and local treatment with curative intent. Eur Urol. 2017;71:618-629.

5. Schouten MG, van der Leest M, Pokorny M, et al. Why and where do we miss significant prostate cancer with multi-parametric magnetic resonance imaging followed by magnetic resonance-guided and transrectal ultrasound-guided biopsy in biopsy-naive men? Eur Urol. 2017;71:896-903.

6. Ukimura O, Coleman JA, de la Taille A, et al. Contemporary role of systematic prostate biopsies: indications, techniques, and implications for patient care. Eur Urol. 2013;63:214-230. 
7. Schoots IG, Roobol MJ, Nieboer D, Bangma CH, Steyerberg EW, Hunink MG. Magnetic resonance imaging-targeted biopsy may enhance the diagnostic accuracy of significant prostate cancer detection compared to standard transrectal ultrasound-guided biopsy: a systematic review and meta-analysis. Eur Urol. 2015;68:438-450.

8. Siddiqui MM, Rais-Bahrami S, Turkbey B, et al. Comparison of MR/ultrasound fusion-guided biopsy with ultrasound-guided biopsy for the diagnosis of prostate cancer. JAMA. 2015;313:390-397.

9. Ahmed HU, El-Shater Bosaily A, Brown LC, et al. Diagnostic accuracy of multiparametric MRI and TRUS biopsy in prostate cancer (PROMIS): a paired validating confirmatory study. Lancet. 2017;389:815-822.

10. Kasivisvanathan V, Rannikko AS, Borghi M, et al. MRI-targeted or standard biopsy for prostate-cancer diagnosis. N Engl J Med. 2018;378:1767-1777.

11. De Visschere PJ, Naesens L, Libbrecht L, et al. What kind of prostate cancers do we miss on multiparametric magnetic resonance imaging? Eur Radiol. 2016;26: 1098-1107.

12. Bratan F, Niaf E, Melodelima C, et al. Influence of imaging and histological factors on prostate cancer detection and localisation on multiparametric MRI: a prospective study. Eur Radiol. 2013;23:2019-2029.

13. Afshar-Oromieh A, Avtzi E, Giesel FL, et al. The diagnostic value of PET/CT imaging with the ${ }^{68} \mathrm{Ga}$-labelled PSMA ligand HBED-CC in the diagnosis of recurrent prostate cancer. Eur J Nucl Med Mol Imaging. 2015;42:197-209.

14. Eiber M, Maurer T, Souvatzoglou M, et al. Evaluation of hybrid ${ }^{68} \mathrm{Ga}-\mathrm{PSMA}$ ligand PET/CT in 248 patients with biochemical recurrence after radical prostatectomy. J Nucl Med. 2015;56:668-674.

15. Perera M, Papa N, Christidis D, et al. Sensitivity, specificity, and predictors of positive ${ }^{68} \mathrm{Ga}$-prostate specific membrane antigen positron emission tomography in advanced prostate cancer: a systematic review and meta-analysis. Eur Urol. 2016;70:926-937.

16. Afshar-Oromieh A, Zechmann CM, Malcher A, et al. Comparison of PET imaging with a ${ }^{68} \mathrm{Ga}$-labelled PSMA ligand and ${ }^{18} \mathrm{~F}$-choline-based PET/CT for the diagnosis of recurrent prostate cancer. Eur J Nucl Med Mol Imaging. 2014;41: 11-20.

17. Simopoulos DN, Natarajan S, Jones TA, Fendler WP, Sisk AE Jr, Marks LS. Targeted prostate biopsy using ${ }^{68}$ gallium PSMA-PET/CT for image guidance. Urol Case Rep. 2017;14:11-14.

18. Sachpekidis C, Kopka K, Eder M, et al. ${ }^{68}$ Ga-PSMA-11 dynamic PET/CT imaging in primary prostate cancer. Clin Nucl Med. 2016;41:e473-e479.

19. Rahbar K, Weckesser M, Huss S, et al. Correlation of intraprostatic tumor extent with ${ }^{68} \mathrm{Ga}$-PSMA distribution in patients with prostate cancer. $\mathrm{J} \mathrm{Nucl} \mathrm{Med}$. 2016;57:563-567.

20. Lopci E, Saita A, Lazzeri M, et al. ${ }^{68} \mathrm{Ga}$-PSMA positron emission tomography/ computerized tomography for primary diagnosis of prostate cancer in men with contraindications to or negative multiparametric magnetic resonance imaging: a prospective observational study. J Urol. 2018;200:95-103.

21. Eiber M, Weirich G, Holzapfel K, et al. Simultaneous ${ }^{68}$ Ga-PSMA HBED-CC PET/MRI improves the localization of primary prostate cancer. Eur Urol. 2016;70: 829-836.
22. Zamboglou C, Drendel V, Jilg CA, et al. Comparison of ${ }^{68} \mathrm{Ga}-\mathrm{HBED}-\mathrm{CC}$ PSMAPET/CT and multiparametric MRI for gross tumour volume detection in patients with primary prostate cancer based on slice by slice comparison with histopathology. Theranostics. 2017;7:228-237.

23. Hicks RM, Simko JP, Westphalen AC, et al. Diagnostic accuracy of ${ }^{68}$ Ga-PSMA$11 \mathrm{PET} / \mathrm{MRI}$ compared with multiparametric MRI in the detection of prostate cancer. Radiology. 2018;289:730-737.

24. Park SY, Zacharias C, Harrison C, et al. Gallium 68 PSMA-11 PET/MR imaging in patients with intermediate- or high-risk prostate cancer. Radiology. 2018;288: 495-505.

25. Zhang Q, Wang W, Zhang B, et al. Comparison of free-hand transperineal mpMRI/TRUS fusion-guided biopsy with transperineal 12-core systematic biopsy for the diagnosis of prostate cancer: a single-center prospective study in China. Int Urol Nephrol. 2017;49:439-448.

26. Weinreb JC, Barentsz JO, Choyke PL, et al. PI-RADS prostate imaging: reporting and data system-2015, version 2. Eur Urol. 2016;69:16-40.

27. Zhang Q, Zang S, Zhang C, et al. Comparison of ${ }^{68}$ Ga-PSMA-11 PET-CT with mpMRI for preoperative lymph node staging in patients with intermediate to high-risk prostate cancer. $J$ Transl Med. 2017;15:230.

28. Eiber M, Herrmann K, Calais J, et al. Prostate cancer molecular imaging standardized evaluation (PROMISE): proposed miTNM classification for the interpretation of PSMA-ligand PET/CT. J Nucl Med. 2018;59:469-478.

29. McNeal JE, Haillot O. Patterns of spread of adenocarcinoma in the prostate as related to cancer volume. Prostate. 2001;49:48-57.

30. Lemaitre L, Puech P, Poncelet E, et al. Dynamic contrast-enhanced MRI of anterior prostate cancer: morphometric assessment and correlation with radical prostatectomy findings. Eur Radiol. 2009;19:470-480.

31. Ploussard G, Epstein JI, Montironi R, et al. The contemporary concept of significant versus insignificant prostate cancer. Eur Urol. 2011;60:291-303.

32. Hoeks CM, Schouten MG, Bomers JG, et al. Three-Tesla magnetic resonanceguided prostate biopsy in men with increased prostate-specific antigen and repeated, negative, random, systematic, transrectal ultrasound biopsies: detection of clinically significant prostate cancers. Eur Urol. 2012;62:902-909.

33. Obuchowski NA. Nonparametric analysis of clustered ROC curve data. Biometrics. 1997;53:567-578.

34. Zeger SL, Liang KY. Longitudinal data analysis for discrete and continuous outcomes. Biometrics. 1986;42:121-130.

35. Smith PJ, Hadgu A. Sensitivity and specificity for correlated observations. Stat Med. 1992;11:1503-1509.

36. Genders TS, Spronk S, Stijnen T, Steyerberg EW, Lesaffre E, Hunink MG. Methods for calculating sensitivity and specificity of clustered data: a tutorial. Radiology. 2012;265:910-916.

37. Leening MJ, Vedder MM, Witteman JC, Pencina MJ, Steyerberg EW. Net reclassification improvement: computation, interpretation, and controversies: a literature review and clinician's guide. Ann Intern Med. 2014;160:122-131.

38. Fütterer JJ, Briganti A, De Visschere P, et al. Can clinically significant prostate cancer be detected with multiparametric magnetic resonance imaging? A systematic review of the literature. Eur Urol. 2015;68:1045-1053. 